\title{
A Low Cost Prosthetic Hand using Arduino and Servo Motors
}

\author{
Ms. Humera Tabassum \\ Dept. of Electronics and Instrumentation \\ P.D.A College of Engineering \\ Kalaburagi Karnataka India
}

\author{
Prof. Veena Saraf \\ Assistant Professor, Dept. of E\&C \\ P.D.A College of Engineering \\ Kalaburagi Karnataka India
}

\begin{abstract}
The idea is to change the perception of remote controls for actuating manually operated Robotic-Hand. Well, this paper provides the buttons or joysticks to controlling the complete Robotic Hand by the users hand. In this paper we deal with the design and development of a Five Fingered Robotic Hand (FFRH) using Arduino board, keypad and servo motor. The design of the system is based on a simple, flexible and minimal control strategy. The Robotic Hand has some independent commands for all the five fingers open and close, wrist up and down, base clockwise and counters clockwise, movement of bot, Pick and Place and Home position to move the fingers. Implementation of pick and place operation of the different object using these commands are discussed. The Arduino based human hand replication system is a system that can help and secure the human presence to be put under harmful situations such as radioactive and bio hazardous. The technology can also be helpful in very precise instrumentation workings like a doctor operating a patient by a robot without its own hands. The technology has its many useful applications in the field of robotics, surgical operations, humanoid robots, etc.
\end{abstract}

\section{INTRODUCTION}

This paper deals with the Design and Implementation of "A Low Cost Prosthetic Hand using Servo Motors". The system design is has 3 parts namely: Keypad Part, Robotic Hand and Platform. It is basically an Arduino based Robotic Hand system which controls a Robotic Hand using keypad using a, small and low-cost, micro switches. The Robotic Hand is mounted over a movable platform which is controlled by another hand.

With 3D printed prosthetics gaining popularity with the advent of consumer level 3D printers, practical applications of these prosthetics have also been increasing. Online open source development has allowed for printable hand models to be downloaded for free within minutes from websites such as Thingiverse, a free, open source 3D modelling site. Contrary to traditional prosthetics, which often cost tens of thousands of dollars1 and are usually unaffordable to many, these alternatives provide a relatively inexpensive option to the public. This allows for less expensive, yet effective prosthetics to be available to modern consumers, with increased personalization for the user at a speed unachievable by conventional methods.

Besides cost, another prevalent issue in high-level prosthetics is ease of control. The most common control system in use is electromyography, a medical technique in which electrical signals from the remaining muscles in an amputee's forearm are read by a device attached to the muscle and are mimicked by the prosthetic. While people without any functional forearm muscles and even those with intact forearm muscles can use this approach, electromyography can be difficult to use and is often imprecise. Therefore, developing a 3D printable hand that is easy to assemble and control in a nonprofessional setting with commonplace products such as Smartphone's is vital for the average amputee. Smartphone based controls are more portable and convenient than traditional myoelectric or motion sensing control systems. Furthermore, the functionality and performance of consumer level robotic hands have advanced significantly in the past several years.

Developments in the 3D printing industry have led to numerous designs of modelled hands from users all around the world, which the public can access online. 3D printed prosthetics are further advantageous in that they are ideal for children and teenagers; when one hand becomes too small, another can be readily printed in a larger size and reintegrated into the control system.

The merits of our Research:

1. It helps the people who have lost one of their hands or if it is paralysed.

2. Low cost. The whole work can completed under 15,000 rupees, which makes the robotic hand very affordable in comparisons to its present contemporary robotic hand proposal.

3. Very easily portable. Its is easy to carry the hand around.

4. Light weight 3D printed modules/parts, makes it very easy for the amputees to carry it around with them.

5. Has a lot of scope for modification. Its working can be made completely automatic and wireless. It can be controlled through cloud or Wi-Fi.

\section{PROSTHETIC HAND DESIGN}

\section{A. Materials and Procedures}

The hand is designed using SolidWorks Computer Aided Design (CAD) software. SolidWorks is utilized because of easy to use and operate. The solid model of prosthetic hand design can be exported into Sim Mechanics model for 3D animation and kinematics analysis purposes. The 3D CAD model of the prosthetic hand cover can be seen in Fig. 1. In order to the proposed of prosthetic hand has the same size and weight with the natural human hand, all of mechatronics component has are designed to fit into the hand.

After the 3D model is developed in Solid Works, the model needs to be printed using 3D printer. The hand is made from PLA (Polylactic Acid) material. The material is selected due to lightweight property. The 3D hand model of this prosthetic hand is inspired by Ada Hand from Open 
Bionics. The size of the prosthetic hand is $180 \mathrm{~mm}$ in length, $85 \mathrm{~mm}$ in width, and $50 \mathrm{~mm}$ in thick. The overall mass of the prosthetic hand is 261 grams. The size, shape, and weight of the prosthetic hand approach with the human natural hand. This is very lightweight prosthetic hand, when the user/transradial amputee used it as prosthetic hand, the user can manipulate object grasping task without feels fatigue. The general characteristics comparison of AstoHand v1.0 can be summarized in Table 2. Based on the Table II, the mass of available robotic hand varies from $261 \mathrm{gr}$ to $760 \mathrm{gr}$.

\section{B. Robotic Arm:}

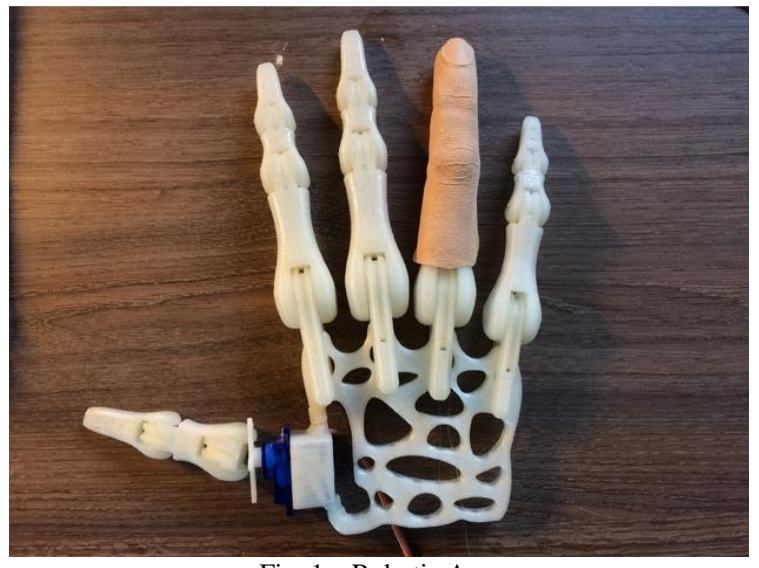

Fig. 1. Robotic Arm

This part of the paper is the real show-stopper. This is the Robotic hand's frame. It can be made from a lot of things like wood, plastic, metal, etc. It is the part that drives the main motive of the project. A 3D printed frame is the most suitable for this paper since it is easy to make, light weight and cheap. Almost any design specification can be met using 3D printing techniques.

1. Other than these main components we need jumper wires for connections.

2. We need a bread board for converging all the connections into one place.

3. A 7.4v $2200 \mathrm{mAh}$ Lithium-Poylmer battery to power the servo motors and 9v Nickel- Cadmium battery for powering the ARDUINO.

4. Fishing nylon wires for the movements of the hand frame.

The servo motor is a linear actuator. There are two types of servo motors, one which rotates $360^{\circ}$ and is continuous and, another one which has a $180^{\circ}$ rotation and is non continuous.

The problem with continuous motor is that it does not move in the backward direction, it is always clockwise or anticlockwise. This is a drawback as the fingers move both front and back. Hence we chose the $180^{\circ}$, non-continuous motor.

\section{Published Specifications of Commercial Hands}

The six hands shown in Figure 1 represent the latest developments in commercial myoelectric hands. While the iLimb (Touch Bionics; Livingston, United Kingdom) and Bebionic (RSL Steeper; Leeds, United Kingdom) hands have received much media attention, the Vincent (Vincent Systems; Weingarten, Germany) and Michelangelo (Otto Bock; Duderstadt, Germany) hands are just becoming available to the public. Therefore, published information on the Vincent and Michelangelo hands is limited.

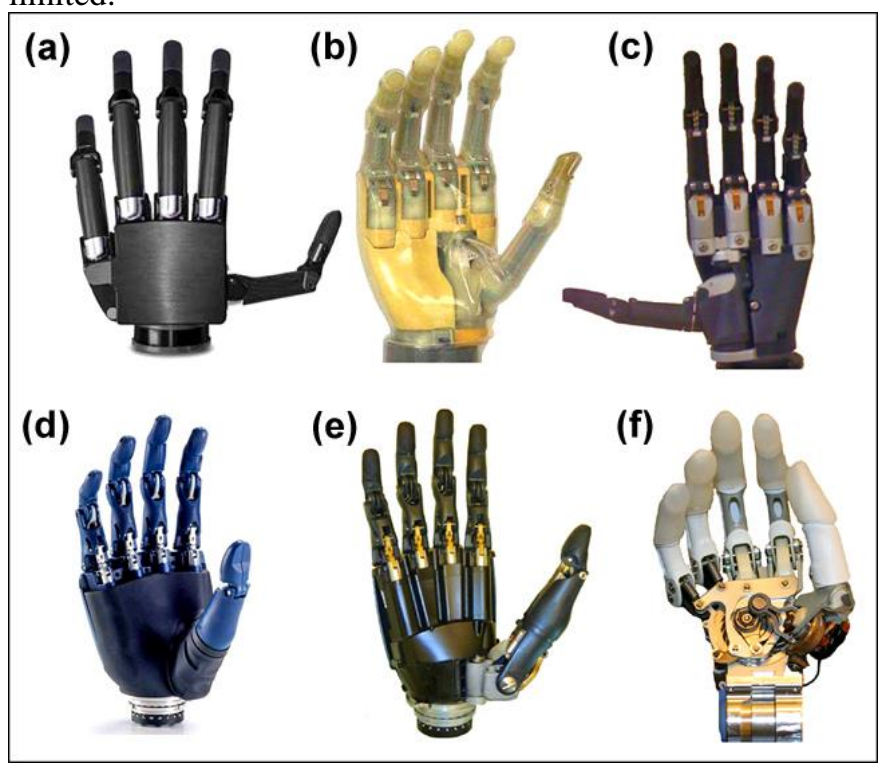

Fig. 2. (a) Vincent hand by Vincent Systems, (b) iLimb hand by Touch Bionics, (c) iLimb Pulse by Touch Bionics, (d) Bebionic hand by RSL Steeper, (e) Bebionic hand v2 by RSL Steeper, and (f) Michelangelo hand by Otto Bock. All hands shown without cosmetic glove.

\section{PROPOSED HARDWARE ARCHITECTURE:}

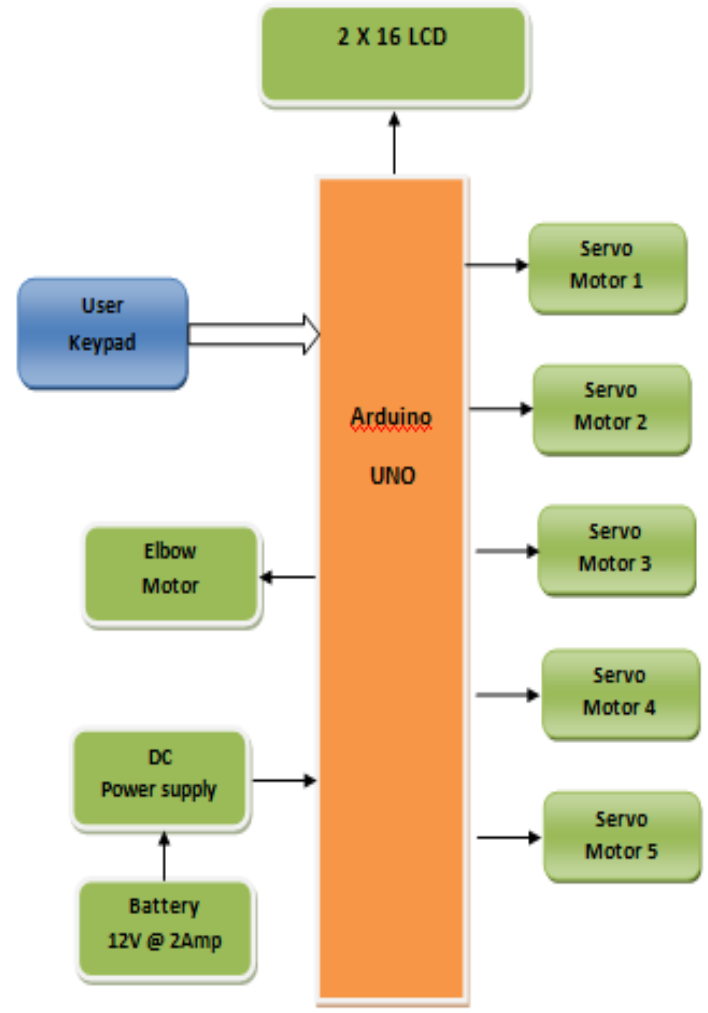

Fig. 3. System block diagram

IV. METHODOLOGY:

This paper represents the step-wise procedure for manufacturing prosthetic robotic arm using 3D printed parts of the robotic hand which includes the four separated parts when we join them it makes the finger of arm and a servo 
motor case, in which we join the servo motor which is connected to the fingers, Arduino Uno, and keypad. The proposed system has switches/keypad as input which is controlled by user's other hand. This project changes the remote controlling criteria of robotic hand. In this paper Arduino board is connected to the input and output that is it controls the whole mechanism of the system through the code or program given to the board and performs the successful functioning of the prosthetic hand. It is very help full to perform daily life activities and it also waste applications I industries where harmful chemicals products are used which can damage the human skin.

The robotic hand consists of five fingers designed using three-dimension 3D printer. Each finger has three degree of freedom (3-DOF) and it is controlled by one servomotor for actuating with angle of rotation from $0^{\circ}$ to $180^{\circ}$. The forward and inverse kinematics for the index figure is derived mathematically for 3-DOF robot finger, and the third order polynomial trajectory motion is simulated to test the robot kinematics by using MATLAB Simulink. The physical tests show that the hand motion serves concisely for catching objects with a maximum distance range for connection of 37 meters. Also, the simulation results show exact inverse kinematic using the equations derived from the forward kinematic. This robotic hand can be applied in the areas where the intervention of humans may cause danger on their health due to chemical, biological and radiological nature.

\section{SOFTWARE DESCRIPTION:}

After hardware connection of each component together we have to put the code in the Arduino board which is the programming module of this project, we have written the code in Arduino language, which we have to load into the programming module then we have to compile the code to check whether the written code have any error or not then if we not get any error we have to upload the code, after uploading the project starts working according to the uploaded code. We have fours software steps in this processing, they are as follows;

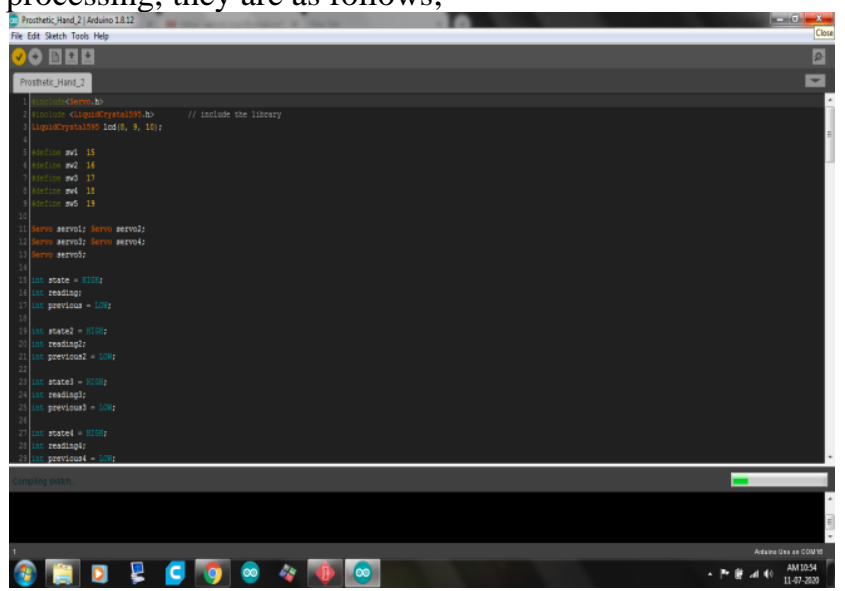

Fig. 4. Compiling

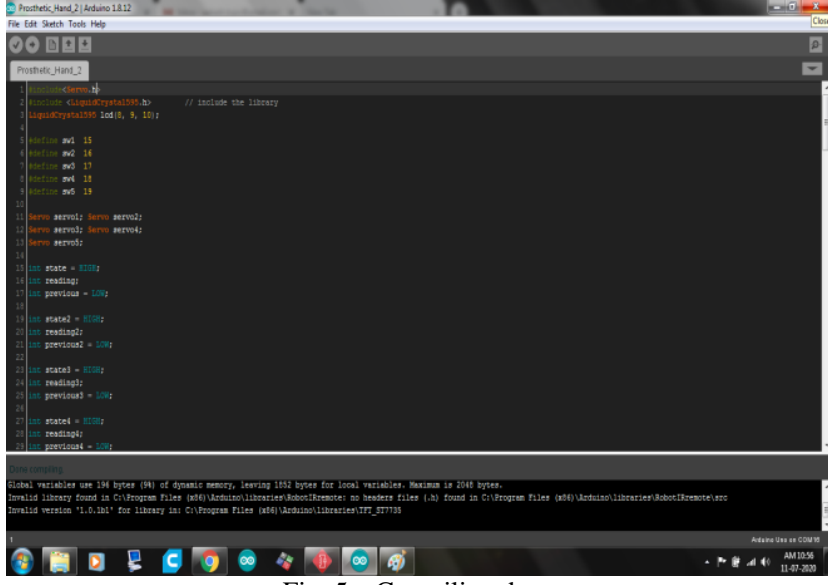

Fig. 5. Compiling done

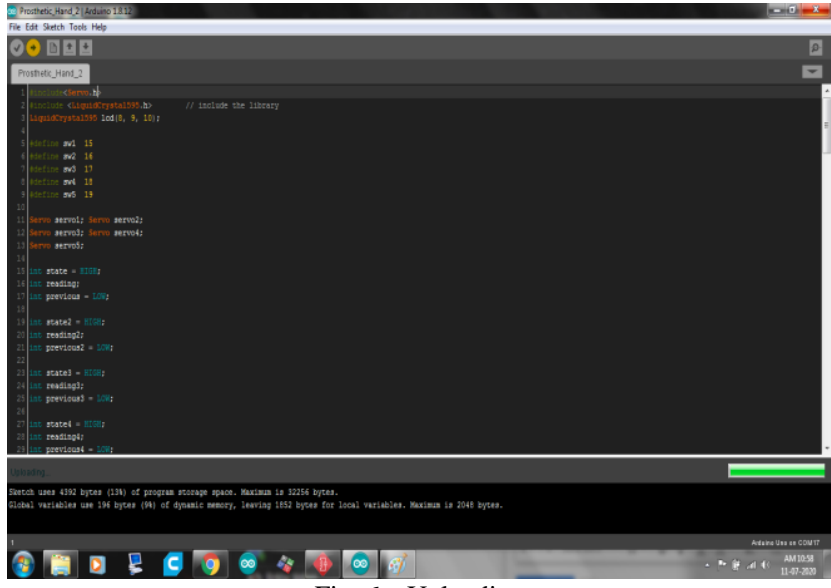

Fig. 6. Uploading

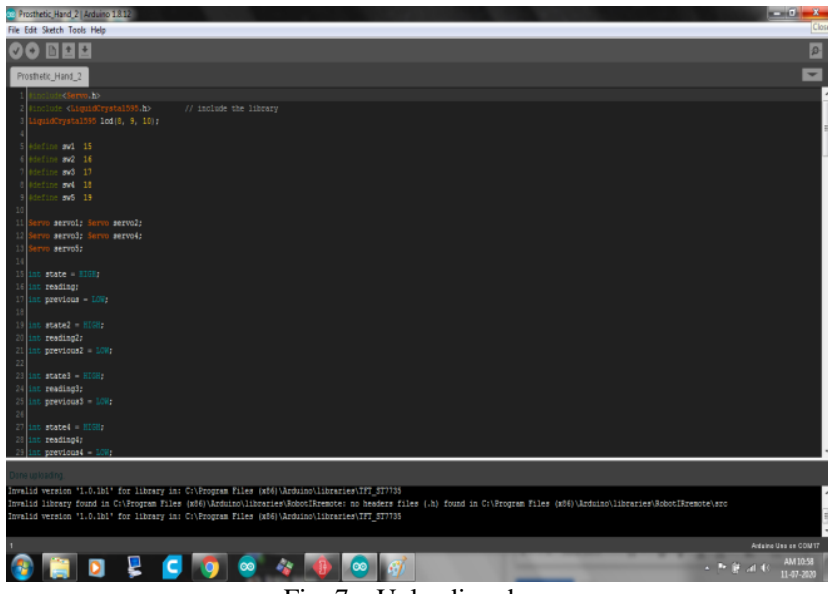

Fig. 7. Uploading done

The above figure 4.14 shows the compiling step of code to check errors or mistakes in the program. The above figure 4.15 shows that after the compilation no error is present in the code and compiling is done. The above Figure 4.16 shows the uploading sketch of code. The above Figure 4.17 shows code is uploaded in the Arduino Uno module. 


\section{EXPERIMENTATION AND RESULTS}

The proposed project is operating completely with battery. The frame of project is made up of 3D-printed parts which is connected with hardware components that are; Arduino Uno, servo motor, keypad, power supply and LCD. This prosthetic Arm is attached to the handicap person after which their life tasks become easy. The assessment of project is conducted using basic function.

Experimental task:

The task includes

1. Opening of arm

2. Closing of arm

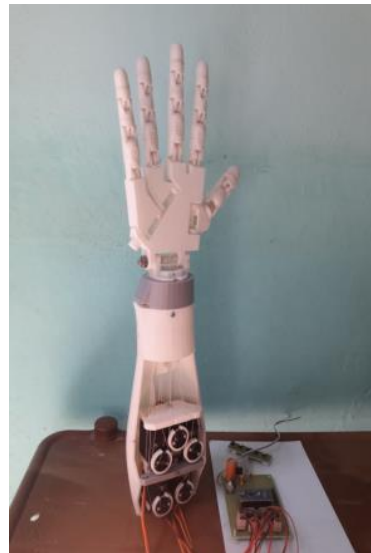

Fig. 8. Opening of arm

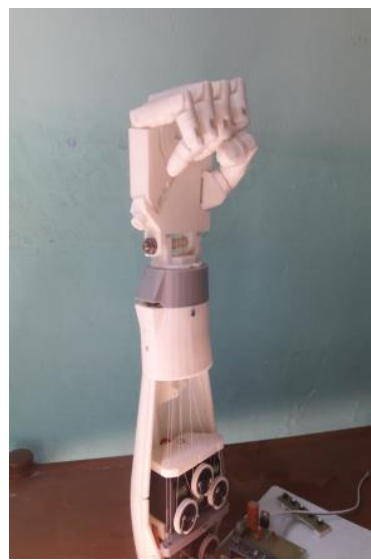

Fig. 9. Closing of arm

\section{CONCLUSIONS}

The proposed of the prosthetic hand is light weight. The servo motors as actuator give the hand lightweight structure and low cost prosthetic hand. This hand can be used as prosthetic hand for transradial prosthesis because of its size and weight which approach the human hand. The prosthetic hand also has seven grip patterns that enable it to do activities of daily living. It works just like a human hand simulation, but with a lesser precision of course. The prosthetic hand just doesn't have applications in helping the physically disabled people, but also has wide applications in manufacture industries, field of aeronautics and space, in mining industries and many more.

\section{ACKNOWLEDGMENT}

I am thankful to my guide, Prof. Veena Saraf for her constant encouragement, guidance and for the continues support in making this work complete.

\section{REFERENCES}

[1] S.M.Mane, R.A.Kambli, F.S.Kazi, N.M.Singh," Hand Motion Recognition From Single Channel Surface EMG Using Wavelet \& Articial Neural Network,"Procesia Computer Science, vol.49, pp.58652015.

[2] M. Ariyanto et al., "Finger movement pattern recognition method using artificial neural network based on electromyography (EMG) sensor," 2015 International Conference on Automation, Cognitive Science, Optics, Micro Electro-Mechanical System, and Information Technology (ICACOMIT), Bandung, 2015, pp. 12- 17.

[3] J Raines, Robotics Hand Development Kit-Ada V1.1 -Datasheet, United Kingdom, Open Bionics, May 2016.

[4] Open Hand Project, Bristol (United Kingdom), Dextrus Hand, 2014.

[5] Vincent Systems, Weingarten (Germany), Vincent Hand, 2014.

[6] Michelangelo operation manual. Duderstadt (Germany): Otto Bock; 2012.

[7] RSL Steeper web site. Leeds (United Kingdom): RSL Steeper; 2013. Available from:http://rslsteeper.com/.

[8] Touch Bionics Inc, Mansfield (MA), Touch Bionics, 2014. Available from: http://www.touchbionics.com/.

[9] [9] Cipriani C, Controzzi M, Carrozza MC. "Objectives, criteria and methods for the design of the SmartHand transradial prosthesis".

[10] Robotica. 2010;28(6):919-27.

[11] Belter JT, Segil JL, Dollar AM, Weir RF. "Mechanical design and performance specifications of anthropomorphic prosthetic hands:

[12] A review".J Rehabil Res Dev.;50(5):599-618, 2013. 